The Army Medical Department has qualified applicants far in excess of its needs, attracted by better pay, well-defined rank, and more satisfactory professional position. Since 1870 more than thirty young medical officers have resigned (three of them to enter the Army Corps)."

A very similar condition of affairs, so far as rank is concerned, now obtains in the British Army. Relative rank has been abolished, and the profession of almost the whole British Empire is justly indignant. And in regard to the condition of affairs in our Naval Medical Corps we may quote from an editorial in the British Medical Journal, of July $\mathbf{2}$ : "Is it a wise and statesmanlike proceeding, all other considerations apart, to leave this wrong without a remedy? There can be but one answer to this question. It is not wise, it is not statesmanlike. Is it good to lower the self-respect of the service? Is it good for the health and efficiency of the most costly army in the world, so to treat its officers as to scare from its ranks the very men a wise Minister should do all he reasonably can to attract into it?"

Membership in the Congress.-The SecretaryGeneral of the Congress is prepared to promptly furnish all persons in other countries applying to him for registration, with a certificate of which the following is a copy:

NINTH INTERNATIONAL MEDICAL CONGRESS. WASHINGTON, D. C., I887.

Office of the Secretary-General:

This Certifies, That...

is a Member of the Ninth International Medical Congress, and (with the members of his family), is entitled to the special rates of transportation to and from the Congress.

JoHN B. HAMILTON, Secretary-General.

By...................

DUTY on EXHIBITS FOR THE CONGRESS. - In another column of this issue of THE JOURNAL will be found an important official letter from the Secretary of the Treasury of the U. S., answering the many questions heretofore asked in reference to how far members of the International Medical Congress to convene in Washington, September 5,1887 , coming from other countries, could bring with them instruments, books, maps, or other materials to be used for illustrations in the work of the Congress, free from revenue taxes. Our foreign exchanges would confer a favor by calling the attention of their readers to the facts stated in the letter.

A Vacancy in the Illinols State Board of
Health.-It has been announced in the daily papers that Dr. George N. Kreider, of Springfield, on the I 8 th inst. sent his resignation to the Governor as a member of the State Board of Health, to take effect as soon as his successor was appointed and qualified. No reasons are given for such resignation.

\section{SPECIAL ARTICLE.}

\section{REPORT ON PASTEUR'S TREATMENT OF HYDROPHOBIA.}

[The report of the committee of the Local Government Board, appointed in April, 1886, to inquire into M. Pasteur's treatment of hydrophobia, was presented to Parliament on June 27. We are indebted The Lancet, of July 2, for the following abstract of the report:]

The report commences by stating that it was found necessary that some of the members of the committee should, together with Mr. Victor Horsley, the secretary, visit Paris so as to obtain information from M. Pasteur himself, to observe his method of treatment, and investigate a considerable number of cases of persons inoculated by him; and, further, that a careful series of experiments should be made by Mr. Horsley on the effects of such inoculation on the lower animals. Mr. Horsley's experiments are stated to entirely confirm M. Pasteur's discovery of a method by which animals may be protected from the infection of rabies. If a dog, rabbit, or other animal be bitten by a rabid dog and die of rabies, a substance can be obtained from its spinal cord which, being inoculated into a healthy dog or other animal, will produce rabies similar to that which would have followed directly from the bite of a rabid animal, or differing only in that the period. of incubation between the inoculation and the appearance of the characteristic symptoms of rabies may be altered. The rabies thus transmitted by inoculation may, by similar inoculations, be transmitted through a succession of rabbits with marked increase of intensity. But the virus in the spinal cord of rabbits that have died of inoculated rabies may be gradually attenuated by drying the cords, so that after a number of days' drying, it may be injected into healthy rabbits or other animals without any danger of producing rabies; and by using on each successive day the virus dried during a period shorter than that used on the previous day an animal may be made almost certainly secure against rabies, whether from a bite or from any method of subcutaneous inoculation; and this protection is proved by the fact that if animals so protected and others not thus protected be bitten by the same rabid animal, none of the first set will die of rabies, and, with rare exceptions, all of the second set will succumb.

It may hence be deemed certain that M. Pasteur has discovered a method of protection from rabies comparable with that which vaccination affords against infection from smallpox. It would be diffcult to over-estimate the importance of the discovery, 